\title{
COORDINATION OF BUS DEPARTURES BY MATHEMATICAL PROGRAMMING
}

This paper deals with coordination of bus arrivals. A quality criterion of this problem is to minimize waiting time of passengers, to make public transport more attractive. One possibility for solving is to increase the number of arrivals with associated investments. Another possibility, without investment, is the coordination of bus arrivals. The latter possibility is analyzed and solved in this paper. Mathematical formulation of this problem leads to a quadratic programming model which is hard to solve. Our approach is based on piecewise linearization of the quadratic objective function. This integer programming model enables to include to the problem some other non-trivial aspects of arrival coordination. These additional aspects are the necessity of safety break compliance and order rearrangement of bus arrivals at a given bus stop. In this paper, integer programming models of the above mentioned problems are presented and the associated numerical experiments are reported to enable comparison of the suggested approaches.

Keywords: Public transport, coordination of bus arrivals, waiting time of passengers, integer programming, free order of objects.

\section{Introduction}

A mathematical programming model of the regular arrival deployment problem was formulated and solved in the several last decades to obtain solution of time coordination of bus arrivals. In this model, regular distribution of time intervals was taken as a quality criterion of searched solution, even if the original objective was to minimize the total waiting time of passengers. Works with time coordination of bus arrivals were published in [1], [2], [3] and [4]. We focused other approach on the non-investment increasing of public transport attractiveness.

In the problem, $n$ arrivals of vehicles at a stop in the designate headway are considered. Let $t_{i}$ be arrival time of vehicle $i$ at the stop. The earliest possible arrival time of the vehicle $i$ is denoted as ai. This time may be postponed at most until the time $a_{i}+c_{i}$, where $c_{i}$ is the maximum possible shift of arrival at the stop. It is necessary to find such time positions of the individual arrivals so that the total passengers' waiting time is minimal.
Arrival times $t_{0}$ and tn are fixed. The goal is to shift times $t_{i}$ for $i=1, \ldots, n-1$, so that the overall waiting time of passengers in passenger-minutes is minimal. Fig. 1 shows how the waiting time depends on the arrival time distribution in the time headway $\left\langle t_{0}, t_{n}\right\rangle$. The grey area represents the total waiting time of passengers coming to the stop in a given headway and waiting for a bus.

The total waiting time of passengers in the headway $\left\langle t_{0}, t_{n}\right\rangle$ can be expressed as:

$$
\sum_{i=1}^{n} \frac{1}{2} f\left(t_{i}-t_{i-1}\right)^{2}=\frac{1}{2} f \sum_{i=1}^{n} v_{i}^{2}
$$

where we introduce a variable $v_{i}$, which represents the length of time interval between two succeeding arrivals $t_{i-1}$ and $t_{i}$ for $i=1$, $\ldots, n$ on the assumption that passengers arrive at the stop equally, uniformly with intensity $f$, where we assume that $f$.

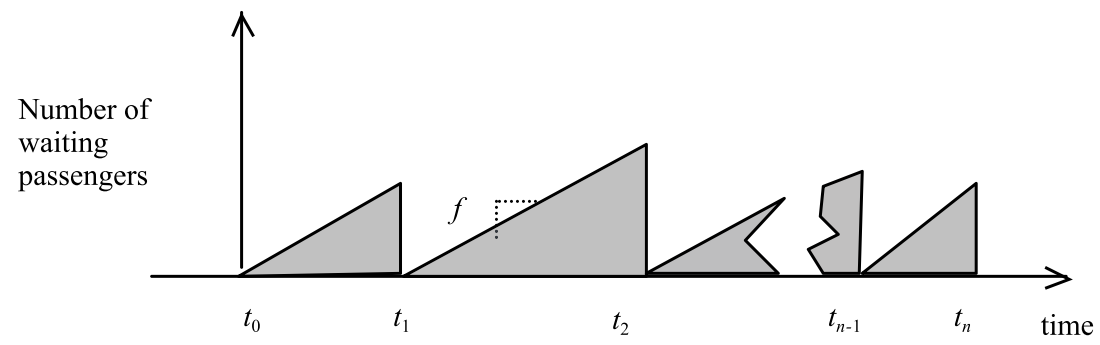

Fig. 1 The waiting time of passengers during headway $\left\langle t_{0}, t_{n}\right\rangle$

\footnotetext{
* Lydia Gabrisova ${ }^{1}$, Petr Kozel ${ }^{2}$

${ }^{1}$ Department of Mathematical Methods, Faculty of Management and Informatics, University of Zilina, Slovakia, E-mail: lydia.gabrisova@fri.uniza.sk,

${ }^{2}$ Department of Mathematical Methods in Economics, Faculty of Economics, Technical University of Ostrava, Czech Republic
} 
We consider here the situation when the stop is serviced by busses, which follow different routes, but a considerable part of their routes is common. We also assume that demanded arrival times, which are most suitable for the passengers using only the common part, are evenly distributed. Then even the passengers, which arrive at the stop accordingly to a time table, loose their time either out of the stop or at the ends of their trips.

\section{Mathematical model of the basic problem}

We introduce variable $x_{i}$ for $i=1, \ldots, n-1$, which models a shift of the arrival time $t_{i}$ from the earliest possible arrival time $a_{i}$. Then the mathematical model of the problem will be as follows:

$$
\text { Minimize } \frac{1}{2} f \sum_{i=1}^{n} v_{i}^{2}
$$

Subject to $x_{1}+a_{1}-t_{0}=v_{1}$

$$
\begin{aligned}
& x_{1}+a_{i}-x_{i-1}-a_{i-1}=v_{i} \\
& \text { for } i=2, \ldots, n-1 \\
& t_{n}-x_{n-1}-a_{n-1}=v_{\mathrm{n}} \\
& v_{i} \geq 0 \text { for } i=1, \ldots, n \\
& x_{i} \leq c_{i} \text { for } i=1, \ldots, n-1 \\
& x_{i} \geq 0 \text { for } i=1, \ldots, n
\end{aligned}
$$

The constraints (3) - (5) determine the gap between two consecutive arrivals as the relation between the values of the variables $v_{i}$ and $x_{i}$. Due to the squares of variables $v_{i}, i=1, \ldots, n$, the objective function (2) is non-linear. In [5] and [6], the similar situation was solved using rearrangement of the original non-linear problem to simpler max-min problem. The following research was focused on search for other approaches, which were published in [7], [8], [9] and [10] together with numerical experiments on real data.

In this paper we will approximate the quadratic non-linear function (2) by a piecewise linear function. This linearization approach has been analyzed in details in [11] and [12].

\section{Arrival deployment problem with the constraints for compliance of safety breaks}

\subsection{Mathematical model of arrival deployment problem}

This section is focused on real problem instances of public transport with the constraints for compliance of safety breaks. The safety breaks are important elements of each vehicle schedule. The safety breaks serve for the relaxation of drivers. It is necessary to place them into the schedule and to take into account that they must not be interrupted. The situation is depicted in Fig. 2 .

There are two alternatives (time windows ) for the possible arrival time of the bus arrival $i$ : either $t_{i} \in\left\langle a_{i}, a_{i}+c_{i}\right\rangle$ or $t_{i} \in\left\langle a_{i}+\right.$ $\left.+c_{i}+s_{i}, a_{i}+r_{i}\right\rangle$, where si is the length of the safety break and $r_{i}$ is the maximal shift of the arrival time $t_{i}$ from $a_{i}$ in the second time window. We introduce these constraints for the compliance of the safety breaks into the mathematical model (2) - (8). As for the objective function, we use a linearization approach for linearization of (2).

The mathematical model of the problem with the constraints for compliance of the safety breaks will be as follows:

$$
\begin{aligned}
& \text { Minimize } \quad \frac{1}{2} f \sum_{i=1}^{n} \sum_{j=1}^{m(i)}(2 j-1) \cdot u_{i j} \\
& \text { Subject to } \quad x_{1}+a_{1}-t_{0}=\sum_{j=1}^{m(1)} u_{1 j} \\
& x_{i}+a_{i}-x_{i-1}-a_{i-1}=\sum_{j=1}^{m(i)} u_{i j} \text { for } i=2, \ldots, n-1 \\
& t_{n}-x_{n-1}-a_{n-1}=\sum_{j=1}^{m(n)} u_{n j} \\
& x_{i} \geq\left(c_{i}+s_{i}\right) y_{i} \quad \text { for } i=1, \ldots, n-1 \\
& x_{i} \leq c_{i}+\left(r_{i}-s_{i}\right) y_{i} \quad \text { for } i=1, \ldots, n-1 \\
& x_{i} \geq 0 \text { for } i=1, \ldots, n-1 \\
& u_{i j} \geq 0 \text { for } i=1, \ldots, n, j=1, \ldots, m(i)
\end{aligned}
$$

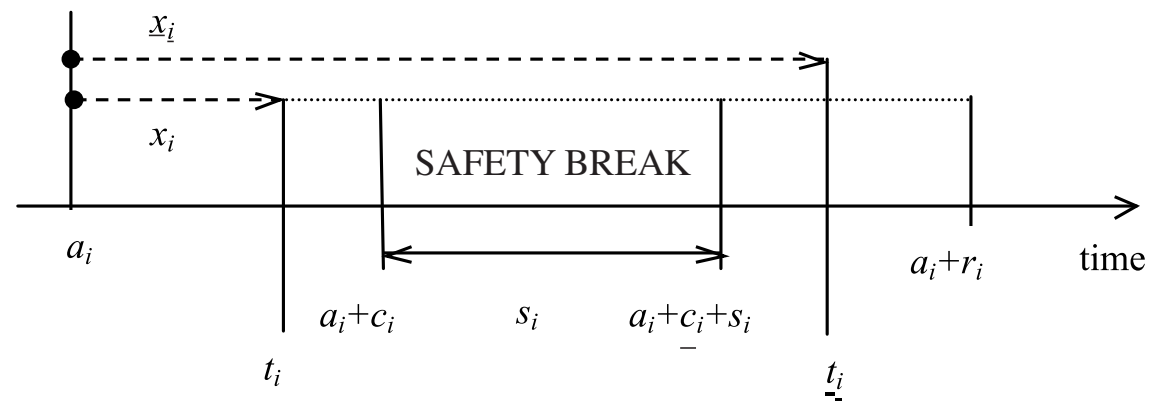

Fig. 2 The possible arrival times ti and ti in regard to position of the safety break 


$$
\begin{aligned}
& u_{i j} \leq 0 \text { for } i=1, \ldots, n, j=1, \ldots, m(i) \\
& y_{i} \in\{0,1\} \text { for } i=1, \ldots, n
\end{aligned}
$$

We approximate the quadratic function (2) by a piecewise linear function (9) by introducing new variables $u_{i j}$ for $j=1, \ldots, m(i)$, $i=1, \ldots, n$ subject to $\sum_{j=1}^{m(i)} u_{i j}=v_{i}$ for $i=1, \ldots, n$.

The constant $m(i)$ for $i=1, \ldots, n$ denotes the number of dividing points of the quadratic function approximation. We consider that each of the time intervals is divided into single minutes. If all the time constants are given in minutes, then we can model these constants as:

$$
\begin{aligned}
& m(1)=a_{1}+r_{1}-t_{0} \\
& m(i)=a_{i}+r_{i}-a_{i-1} \text { for } i=2, \ldots, n-1 \\
& m(n)=t_{n}-a_{n-1} .
\end{aligned}
$$

The constraints (13) - (14) ensure the compliance of safety breaks. For this purpose, we introduce zero-one variables $y_{i}$ to the constraint so that either $x_{i} \leq r_{i}$ and $x_{i} \geq c_{i}+s_{i}$ for $y_{i}=1$ or $x_{i} \leq c_{i}$ and $x_{i} \geq 0$ for $y_{i}=0$ hold.

Note, that even if model (9) - (18) contains more constraints than model (2) - (8), the set of feasible solutions of the problem (9) - (18) is bigger than the solution set of the basic problem.

\subsection{Numerical experiments with the constraints for compliance of safety breaks}

As the optimal solution does not depend on a concrete value of the constant $f$, we put $f=1$ passenger per minute in the following experiments.

We present here a series of numerical experiments by IP-Solver for 8 real problems of the public transport in the selected area of the Czech Republic. In Table 1 we show the comparison of the results of linearized basic problem (2) - (8) and the problem (9)
- (18) with the constraints for the compliance of the safety breaks. The benchmark criterion for the comparison is the total waiting time of passengers in [passenger-minutes], which is the value of the objective function (2). The "default state" mentioned in Table 1 denotes the current deployment of arrivals the running transportation system.

The row "The safety breaks Offered/Used" denotes number of bus arrivals which can use the system of two time windows (separated by a safety break, which is depicted in Fig. 2). The value (used) is number of bus arrivals, which were situated into the second time window $t_{i} \in\left\langle a_{i}+c_{i}+s_{i}, a_{i}+r_{i}\right\rangle$. Usage of the second time window is followed by better value of the objective function.

\section{Problem of time coordination with free order of arrivals at a single stop}

This section is focused on time coordination with a fixed order of bus arrivals. Now, we deal with the problem of time coordination with a free order of bus arrivals. On the contrary to the previous problems in this case a rearrangement of order of bus arrivals at a given bus stop is possible. The associated research is published in [13] together with results of preliminary numerical experiments on real data.

The coordination of arrival times $t_{i}$ and $t_{k}$ are given by two alternatives for the possible order of the bus arrival $i$ and $k$. The examples are illustrated in Figs. 3 and 4.

\subsection{Mathematical model of time coordination with a free order of arrivals}

We introduce zero-one variables wik for $i=0, \ldots, n-1, k=1$, ..., $n$, which model whether the bus arrival $i$ directly precedes the bus arrival $k$ or not. These variables are defined only for pairs ( $i$, $k$ ) of bus arrivals where the direct preceding is possible. Thus

$w_{0 k}$ is defined for $k=1, \ldots, n-1$,

because the fixed arrival 0 directly precedes each free arrival $k$, win is defined for $i=1, \ldots, n-1$,

Comparison of results of the linearized basic problem (2) - (8) and the problem (9) - (18) with the constraints for the compliance of the safety breaks

\begin{tabular}{|c|c|c|c|c|c|c|c|c|c|}
\hline The code of problem & 1 & 2 & 3 & 4 & 5 & 6 & 7 & 8 \\
\hline Number of arrivals & 15 & 19 & 12 & 9 & 9 & 9 & 23 & 9 \\
\hline The safety breaks Offered/Used & $3 / 0$ & $3 / 1$ & $4 / 0$ & $2 / 2$ & $3 / 3$ & $2 / 1$ & $5 / 0$ & $2 / 2$ \\
\hline The variants of solution & \multicolumn{7}{|c|}{ The total waiting time of passengers [passenger-minutes] } \\
\hline $\begin{array}{c}\text { The default state } \\
\text { The linearized basic problem }\end{array}$ & 5508 & 6345.5 & 5628 & 9047.5 & 5250.5 & 5618 & 5460 & 5166 \\
\hline $\begin{array}{c}\text { The problem with the constraints for the } \\
\text { compliance of the safety breaks }\end{array}$ & 5125 & 5319.5 & 4327 & 7837.5 & 4893.5 & 4694 & 4975 & 4464 \\
\hline
\end{tabular}




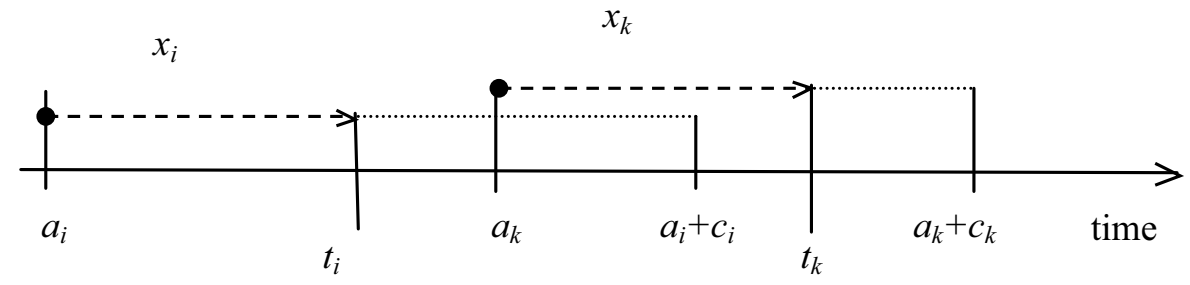

Fig. 3 The possible arrival times $t_{i}$ and $t_{k}$, when ti precedes $t_{k}$

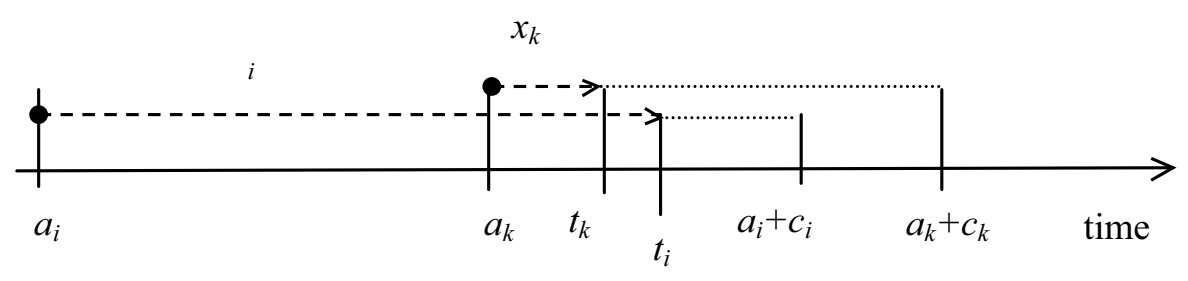

Fig. 4 The possible arrival times $t_{i}$ and $t_{k}$, when $t_{k}$ precedes $t_{i}$

because bus arrival $i$ directly precedes the fixed arrival $n$, $w_{i k}$ is defined for $i=1, \ldots, n-1, k=1, \ldots, n-1, i \neq k$ subject to $a_{i}<a_{k}+c_{k}$.

To make the following model more concise, we introduce a Boolean function exists $\left(w_{i k}\right)$ according to (19) which takes the value of true if a variable $w_{i k}$ is defined and the value of false otherwise. Then the mathematical model of the problem with a free order of arrivals will be as follows:

Minimize $\frac{1}{2} f \sum_{i=1}^{n} \sum_{j=1}^{m(i)}(2 j-1) \cdot u_{i j}$

Subject to $x_{k}+a_{k}-t_{0} \leq \sum_{j=1}^{m(1)} u_{k, j}+\left(t_{n}-t_{0}\right) \cdot\left(1-w_{0 k}\right)$

for $k=1, \ldots, n-1$

$x_{k}+a_{k}-t_{0} \geq \sum_{j=1}^{m(1)} u_{k, j}-\left(t_{n}-t_{0}\right) \cdot\left(1-w_{0 k}\right)$

for $k=1, \ldots, n-1$

$x_{k}+a_{k}-x_{i}-a_{i} \leq \sum_{j=1}^{m(1)} u_{k, j}+\left(t_{n}-t_{0}\right) \cdot\left(1-w_{i k}\right)$

for $i=1, \ldots, n-1 ; k=1, \ldots, n-1 ; \operatorname{exist}\left(w_{i k}\right)$

$x_{k}+a_{k}-x_{i}-a_{i} \geq \sum_{j=1}^{m(1)} u_{k, j}-\left(t_{n}-t_{0}\right) \cdot\left(1-w_{i k}\right)$

for $i=1, \ldots, n-1 ; k=1, \ldots, n-1 ; \operatorname{exist}\left(w_{i k}\right)$ $t_{n}-x_{i}-a_{i} \leq \sum_{j=1}^{m(n)} u_{n, j}+\left(t_{n}-t_{0}\right) \cdot\left(1-w_{i n}\right)$

for $i=1, \ldots, n-1$

$t_{n}-x_{i}-a_{i} \geq \sum_{j=1}^{m(n)} u_{n, j}-\left(t_{n}-t_{0}\right) \cdot\left(1-w_{i n}\right)$

for $i=1, \ldots, n-1$

$\sum_{i=0}^{n-1} w_{i k}=1 \quad$ for $k=1, \ldots, n ; \operatorname{exist}\left(w_{i k}\right)$

$\sum_{k=1}^{n} w_{i k}=1 \quad$ for $i=0, \ldots, n-1 ; \operatorname{exist}\left(w_{i k}\right)$

$x_{i} \geq 0 \quad$ for $\quad i=1, \ldots, n-1$

$x_{i} \leq c_{i} \quad$ for $\quad i=1, \ldots, n-1$

$u_{i j} \geq 0 \quad$ for $\quad i=1, \ldots, n ; j=1, \ldots, m(i)$

$u_{i j} \leq 1 \quad$ for $\quad i=1, \ldots, n ; j=1, \ldots, m(i)$

$w_{i k} \in\{0,1\}$ for $i=1, \ldots, n-1 ; k=1, \ldots, n-1$;

$\operatorname{exist}\left(w_{i k}\right)$

We approximate the quadratic function (2) by a piecewise linear function (20) as mentioned in section 3 . In this case the constant $m(i)$ for $i=1, \ldots, n$ denotes the number of dividing points of the approximation of the quadratic function (2). We consider that each of the time intervals is also divided into single minutes. Then we can model these constants as: 


$$
\begin{aligned}
& m(1)=a_{1}+c_{1}-t_{0}, \\
& m(i)=a_{i}+c_{i}-t_{0} \text { for } i=2, \ldots, n-1, \\
& m(n)=t_{n}-t_{0} .
\end{aligned}
$$

The constraints (21) - (26) cause that if $w_{i k}=1$ for some pair $(i, k)$, then the gap between two succeeding arrivals $t_{i}$ and $t_{k}$ is equal to the sum of $u_{k j}$ for $j=1, \ldots, m(i)$, which models the time between two succeeding arrivals. If $w_{i k}=0$ holds, then the associated constraints are relaxed by suitable value of $t_{n}-t_{0}$. The constraints (27) assure that exactly one arrival $i$ precedes arrival $k$. The constraints (28) assure that exactly one arrival $i$ precedes arrival $k$. The output of the problem (20) - (33) solving is formed by $n+1$ values $w_{i k}$ for $w_{i k}=1$, which define a new rearrangement of the order of arrivals.

\subsection{Numerical experiments for time coordination with a free order of bus arrivals}

We present here a series of numerical experiments performed on IP-Solver for 16 real problems of the public transport in the selected area of the Czech Republic. In Tables 2 and 3, we show the comparison of results of the linearized basic problem (2) - (8) and the problem (20) - (33) with a free order of arrivals. The benchmark criterion for the comparison is the total waiting time of passengers in [passenger-minutes], which is the value of the objective function (2).

In Table 4 we show the comparison of results of one example - the code 8 of the public transport in the area of Frydek Mistek - Dobra for 9 arrivals. We show output of the gap between two arrivals in [minutes] and the total waiting time of passengers in [passenger-minutes] for the linearized basic problem (2) - (8) and the problem (20) - (33) with a free order of arrivals. In this case, the result of the problem with a free order of bus arrivals designs a new rearrangement of the order of arrivals: $0,2,1,4,3,5,6,7,8,9$.

\section{Conclusion}

The paper was focused on the non-investment increasing of public transport attractiveness. The mathematical model with constraints for the compliance of safety breaks and mathematical model

Comparison of results of the linearized basic problem (2) - (8) and the problem (20) - (33) with free order of arrivals

Table 2

\begin{tabular}{|c|c|c|c|c|c|c|c|c|c|}
\hline The code of problem & 1 & 2 & 3 & 4 & 5 & 6 & 7 & 8 \\
\hline Number of arrivals & 15 & 19 & 12 & 9 & 9 & 9 & 23 & 9 \\
\hline The variants of solution & \multicolumn{7}{|c|}{ The total waiting time of passengers [passenger-minutes] } \\
\hline The default state & 5508 & 6345.5 & 5628 & 9047.5 & 5250.5 & 5618 & 5460 & 5166 \\
\hline The linearized basic problem & 5125 & 5319.5 & 4327 & 7837.5 & 4893.5 & 4694 & 4975 & 4464 \\
\hline The problem with free order of arrival & 5036 & 5319.5 & 4326 & 7837.5 & 4731.5 & 4694 & 4961 & 4191 \\
\hline
\end{tabular}

Comparison of results of the linearized basic problem (2) - (8) and the problem (20) - (33)with free order of arrivals

\begin{tabular}{|c|c|c|c|c|c|c|c|c|c|}
\hline The code of problem & 9 & 10 & 11 & 12 & 13 & 14 & 15 & 16 \\
\hline Number of arrivals & 10 & 9 & 10 & 10 & 9 & 5 & 10 & 9 \\
\hline The variants of solution & \multicolumn{7}{|c|}{ The total waiting time of passengers [passenger-minutes] } \\
\hline The default state & 4536 & 6634 & 9923.5 & 9529 & 8282 & 21711.5 & 8007 & 2131 \\
\hline The linearized basic problem & 3557 & 4169 & 8945.5 & 6525 & 5837 & 18029.5 & 6930 & 2084 \\
\hline The problem with free order of arrival & 3555 & 3932 & 7144.5 & 6424 & 5835 & 18029.5 & 6872 & 2084 \\
\hline
\end{tabular}

Comparison of results of the linearized basic problem (2) - (8) and the problem (20) - (33)

Table 4 with free order of arrivals for one problem 8

\begin{tabular}{|c|c|c|c|c|c|c|c|c|c|c|}
\hline \multirow{2}{*}{ Problem 8 } & \multicolumn{9}{|c|}{ The gap between two arrivals [minutes] } & $\begin{array}{c}\text { The total waiting time } \\
\text { of passengers [passen- } \\
\text { ger-minutes] }\end{array}$ \\
\hline The variants of solution & $t_{1}-t_{0}$ & $t_{2}-t_{1}$ & $t_{3}-t_{2}$ & $t_{4}-t_{3}$ & $t_{5}-t_{4}$ & $t_{6}-t_{5}$ & $t_{7}-t_{6}$ & $t_{8}-t_{7}$ & $t_{9}-t_{8}$ & 5166 \\
\hline The default state & 13 & 13 & 45 & 10 & 65 & 18 & 22 & 30 & 44 & 4464 \\
\hline The linearized basic problem & 13 & 13 & 43 & 12 & 46 & 30 & 29 & 36 & 38 & 4191 \\
\hline $\begin{array}{c}\text { The problem with free } \\
\text { order of arrival }\end{array}$ & 25 & 8 & 38 & 18 & 38 & 29 & 30 & 36 & 38 & 4 \\
\hline
\end{tabular}


with a free order of arrivals are presented and compared to the basic problem.

The results of the real problems with models (9) - (18) with the constraints for compliance of safety breaks shown in Table 1 are better than the results obtained with the linearized basic problem (2) - (8).

Also the results of real problems (20) - (33) with a free order of arrivals shown in Tables 2 and 3 are better comparing to the linearized basic problem (2) - (8).

Taking into account that all the computational time for the solved problems did not exceed few seconds, we can conclude that the higher complexity of used models brought considerable savings of passengers' waiting time.

\section{Acknowledgement:}

This work was supported by the research grants VEGA 1/0296/12 "Public Service Systems with Fair Access to Service" and APVV-0760-11 "Designing of Fair Service Systems on Transportation Networks".

We would also like to thank to "Centre of excellence for systems and services of intelligent transport" (ITMS 26220120028) for building up the infrastructure which was used.

\section{References}

[1] HAASE, K., DESAULNIERS, G., DESROSIERS, J.: Simultaneous vehicle and crew scheduling in urban mass transit systems. Transportation Science, vol. 35, No. 3, 2001.

[2] BENOIT, L., JIN-KAO, H.: Simultaneous Vehicle and Crew Scheduling for Extra Urban Transports. Proc. of conference IEA/AIE on Industrial, Engineering and Other Applications of Applied Intelligent Systems: New Frontiers in Applied Artificial Intelligence, 2008

[3] PALUCH, S.: A Note to Vehicle Scheduling with Several Bus Types. Proc. of conference on Mathematical Methods in Economics, Part. II., 2011.

[4] BALL, M., BODIN, L., DIAL, R.: A Matching Based Heuristic for Scheduling Mass Transit Crews and Vehicles. J. of Transportation Science, vol. 17, No. 1, 1983.

[5] CERNY, J., KLUVANEK, P.: Basis of Mathematical Transport Theory. VEDA, Bratislava, 1991, (in Slovak).

[6] JANACEK, J.: Mathematical Programming. EDIS, Zilina, 2003, (in Czech).

[7] JANACEK, J., KOHANI, M.: Waiting Time Optimization with IP-Solver. Communications - Scientific Letters of the University of Zilina, No. 3a, 2010.

[8] JANACEK, J., SIRC, J.: The Column Generation Technique for Public Transport Line Planning by IP-Solver. Communications Scientific Letters of the University of Zilina, No. 3a, 2010.

[9] KOHANI, M., MARTON, P.: Methods and Techniques for Design of Effective and Competitive Single Wagon Load Transportation. Communications - Scientific Letters of the University of Zilina, No. 3, 2009.

[10] KOZEL, P., GABRISOVA, L.: Minimization of Passengers Waiting Time by Vehicle Schedule Adjustment. Proc. of intern. conference Transcom 2011, University of Zilina, 2011, pp. 103-106.

[11] GABRISOVA, L.: Accuracy of Solution of some Non-linear Problems by a Piecewise Linear Approximation. Proc. of workshop Problems of Discrete Optimization for Transport, University of Pardubice, 2010, (in Slovak).

[12] GABRISOVA, L., KOZEL, P.: Accuracy of Linear Approximation Used in Non-linear Problem Optimization. J. of Information, Control and Management Systems of the University of Zilina, vol 8, No. 4, 2010.

[13] JANACEK, J., GABRISOVA, L.: The Solution of Some Problems with Free Order of Objects with IP-Solver (in Czech). Proc. of workshop Problems of Discrete Optimization for Transport, University of Pardubice, 2012. 